\title{
Riding Comfort Simulation with Air Ride Seat for Heavy Duty Vehicle
}

\author{
Hyung Yun Choi ${ }^{1}$ Whe-Ro Lee ${ }^{1}$ Jong-Chan Park ${ }^{2}$ Kee-Young Yang ${ }^{2}$ \\ ${ }^{1}$ HongIk University, Korea, hychoi@ hongik.ac.kr, wirolee@gmail.com \\ ${ }^{2}$ Hyundai Motor Company, Korea, \{impactpart, yky\}@ hyundai.com
}

\begin{abstract}
The design of driver's seat suspension of the commercial truck differs from the one of the passenger car. The vibration and the structural characteristics of the suspension are consequently quite different. Unlike passenger cars, the vibration frequency of commercial truck suspensions is considerably low at 1 to $3 \mathrm{~Hz}$. (Mayton, 2006). The truck seat has an air ride seat. The structural design of air ride seat at heavy-duty vehicle includes serial and parallel combinations of the shock absorber, air spring, and PU foam pad to achieve a good vibration damping. The 1D lumped network solution is an effective design tool with the multi-physical subcomponents. And this also enables a direct coupling into the system modeling of the vehicle body for an optimal calibration of engineering parameters taking the relevant dynamic performance of neighboring parts into account. The mechanical characteristics of each component and their assembly were identified for the 1D modeling. The result of validation and verification of the proposing 1D model of the air ride seat is also introduced.
\end{abstract}

Keywords: Air spring, Seat comfort, ISO 2631

\section{Introduction}

The vehicle seat is one of the important components contributing to the crash safety and riding comfort of the occupant. The design of seat frame requires a high strength and stiffness for the better mechanical performance in a crash situation. In a meanwhile, the upholstery of the seat being in a precise contact with a human occupant needs to utilize sufficiently viscoelastic materials with the good tangent of delta such as lowdensity polyurethane foam for both quasi-static and dynamic supports. Unlike passenger vehicles that mainly drive over the flat road, trucks occasionally undergo a driving over the rugged or even unpaved road. It brings more vigorous oscillation to the truck body. And the cabin has extra pitching motion because of the cabin suspension. It adversely affects the kinematics of occupant at a raised seat position in particular. To suppress the low-frequency oscillation, air suspension is mostly adopted in a seat design for the heavy duty vehicle. The pneumatic pressure actuator which is especially available at the commercial heavy-duty vehicle enables to add the air cell into the seat suspension design for a better isolation of the vibration. The air cell is commonly used also in cabin and chassis suspensions of the heavy-duty vehicle.

Extensive series of lab test has been conducted to characterize the mechanical behavior of major components such as hydraulic damper and pneumatic cylinder, i.e., the air cell unit in the air ride seat. A sine sweep excitation signal was applied to the seat system to measure a transmissibility on a $75 \mathrm{kgf}$ dead weight. This paper introduces the $1 \mathrm{D}$ modeling process of air ride seat system, the mechanical characteristics, and its validation result.

The verification of the $1 \mathrm{D}$ modeling scheme, the other "V" in Verification \& Validation process for a riding comfort virtual simulation is also provided with the assessment of the associated riding comfort Index, ISO 2631 "Evaluation of human exposure to whole-body vibration". The ISO2631 provides an indicator for riding comfort and also evaluates riding quality from simulation results (Yoo, 2006). Since this application is a part of feasibility studies and so a simple rigid body mass model was employed as a surrogate human model. The adaptation of the biofidelic human body model is still in progress and remains as our future challenge.

\section{System modeling of air ride seat}

There are three major parts in an air ride seat design, air spring, shock absorber and PU foam pad to isolate the occupant from the vibration. Air spring and shock absorber aim to counteract jerk type impulse loadings while the PU foam pad is laid for attenuation of the oscillatory pulse in $3-10 \mathrm{~Hz}$ transferring to the occupant. The 1D modeling process of air ride seat system consists of the selection of appropriate model libraries to represent subsystems and the calibration of necessary model parameters. An air ride seat designed for the driver side of a large truck (curb weight $9400 \mathrm{~kg}$ ) was selected and SimulationX (ESI ITI) was adopted for the system simulation software 


\subsection{Seat frame structure}

The dynamic mass properties of the frame structure were calculated from the CAD geometry as shown in Figure 1 . The underbody in frame structure containing air spring and the shock absorber is designed with $\mathrm{X}$ link such that the seat cushion can move freely in the vertical direction, up and down. Figure 2 shows 5 rotational joints and 2 translational joints in the underbody frame X-link structure.

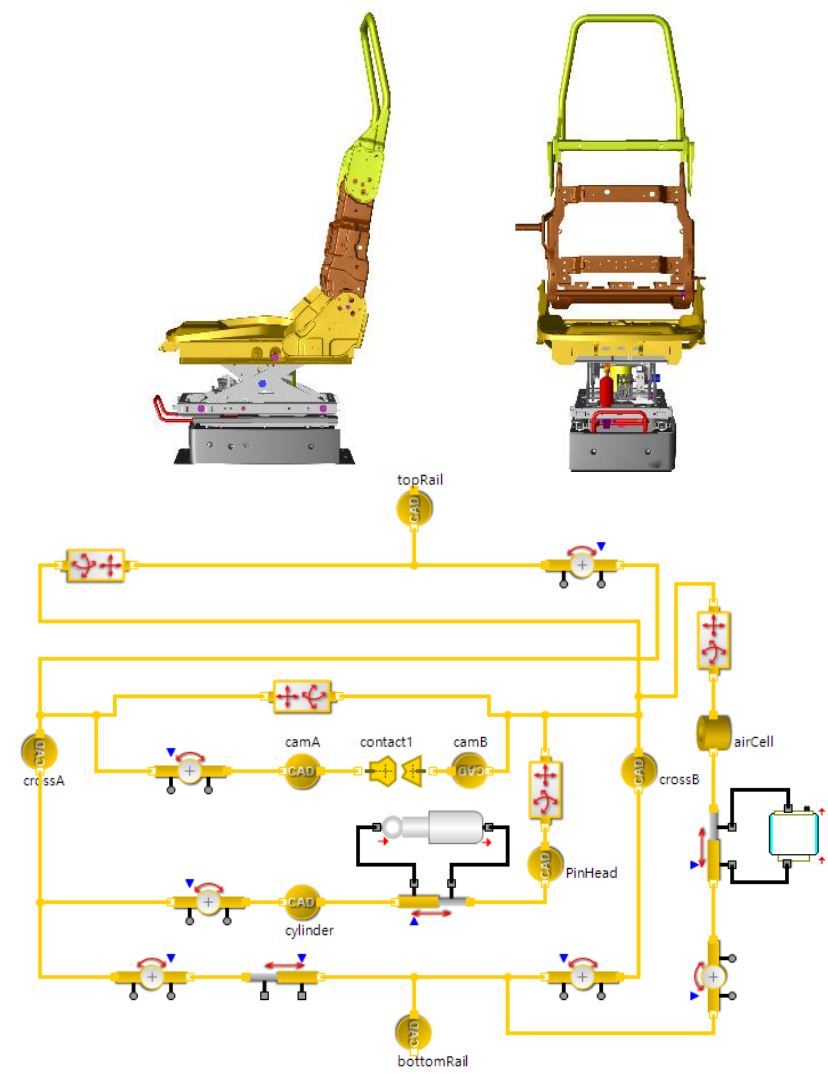

Figure 1. 3D CAD (top) and Diagram (bottom) views of air ride seat frame

\subsection{Air spring modeling}

The stiffness of the air spring $\left(k_{s}\right)$ is expressed as in the following equation (Liu, 2008)

$$
\begin{gathered}
k_{s}=\frac{d F}{d x}=A_{e} \frac{d\left(P-P_{a}\right)}{d x}+\left(P-P_{a}\right) \frac{d A_{e}}{d x} \\
F=\int\left(A_{e} \frac{d\left(P-P_{a}\right)}{d x}+\left(P-P_{a}\right) \frac{d A_{e}}{d x}\right) d x
\end{gathered}
$$

Where

$k_{S}$ : Stiffness of air spring

$F$ : Cross section force

$\mathrm{x}$ : Stroke

$P$ : Air pressure

$P a$ : Ambient air pressure

$A e$ : Initial cross section area

Figure 3 shows the pressure and effective cross section area changes with axial compression of the air spring unit. The abrupt slope increase of force profile

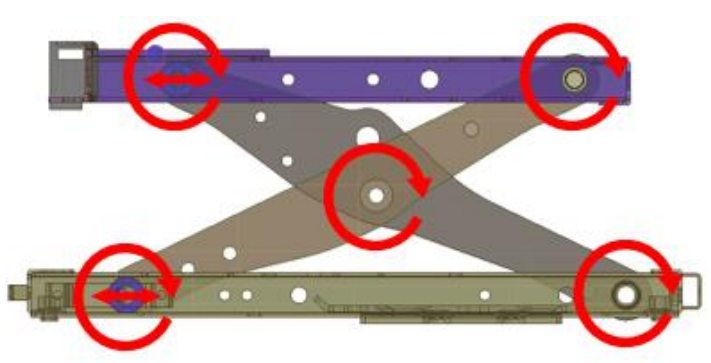

Figure 2. Joints with free DOF in under body frame structure

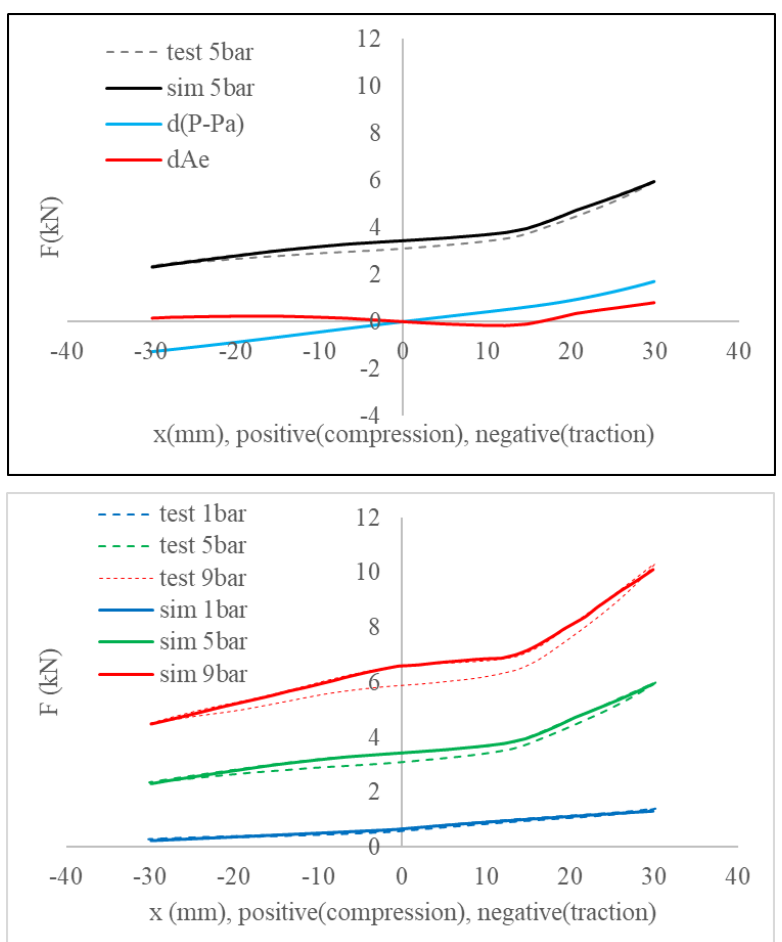

Figure 3. The air spring force is determined by terms of pressure and effective cross-sectional area

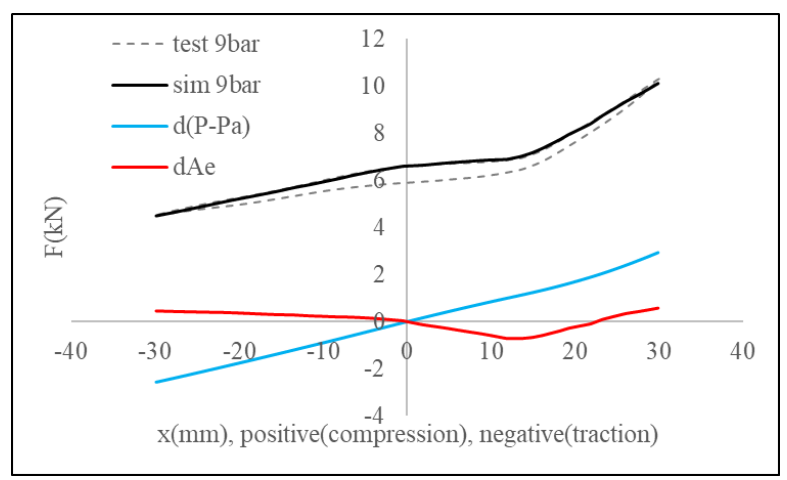

Figure 4. Comparison of force-displacement of air spring unit between test and simulation at various air pressure levels (1-9 bar)

around $10 \mathrm{~mm}$ compression distance occurs due to the change of cross-section area in the air-cell. This nonlinear behavior of the compression stiffness of air spring is also based on the ideal gas equation, the relationship between temperature, pressure, and volume. The 
temperature of the air in the cell is assumed constant. The compression force is determined from the effective cross-section area and the pressure of the cell at a given stroke.

Air spring shows hysteresis, different forcedisplacement paths at loading and unloading phases, which becomes more distinctive at higher air pressure level as shown in Figure 4. The air spring element in SimulationX model library was adopted. Those modeling parameters, i.e., characteristics of pressure and volume to axial force relationships were calibrated from measurements at compression in-vitro test of the air-spring unit. Since the temperature of air in the spring assumed constant, the hysteresis is not adequately presented in the system model.

\subsection{Pneumatic system modeling}

In order to maintain the uniform vertical seat position in a moving vehicle, the air spring has a control logic to inflate and deflate the air cell respectively to raise and lower the seat. The "Auto level valve" (Patent: 10-20030043540) consists of with a control cam and threechannel air tube. It has a kinetic mechanism for gauging the height change of the seat cushion and the system subsequently controls the pressure level in the air spring. Using the pneumatic library in SimulationX, this "Auto level valve" system was integrated as a valve logic in the air ride seat model. The coupling chain of the dynamics of seat height, the rotation of control cam, and the opening/closing of the leveling valve are explicitly simulated in the model as shown in Figure 5.

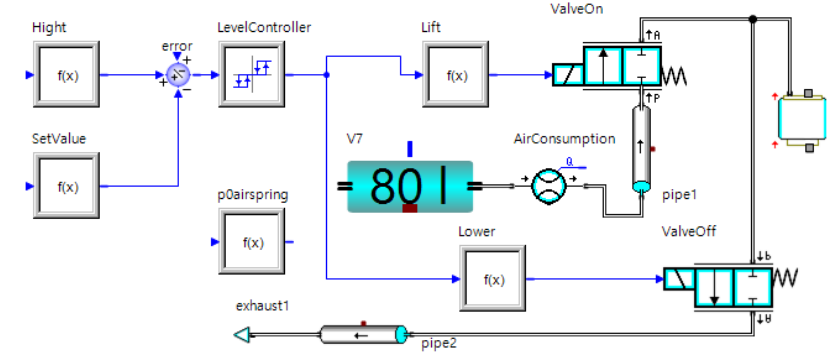

Figure 5. Diagram model of the auto leveling system

\subsection{Shock absorber modeling}

The operating frequency range of the shock absorber is roughly between 1 to $5 \mathrm{~Hz}$, which is an approximate calculation from the typical stroke distance $(20 \pm 10 \mathrm{~mm})$ and the traveling speed $(0.05 \pm 0.03 \mathrm{~m} / \mathrm{s})$ of the seat cushion. The shock absorber of the air ride seat in this study has a design with three-stage, hard, medium, and soft for the damping force control. The measured nonlinear behavior of damping force with the compression speed is shown in Figure 6. This damping characteristics are precisely modeled by the shock absorber element in SimulationX model library.

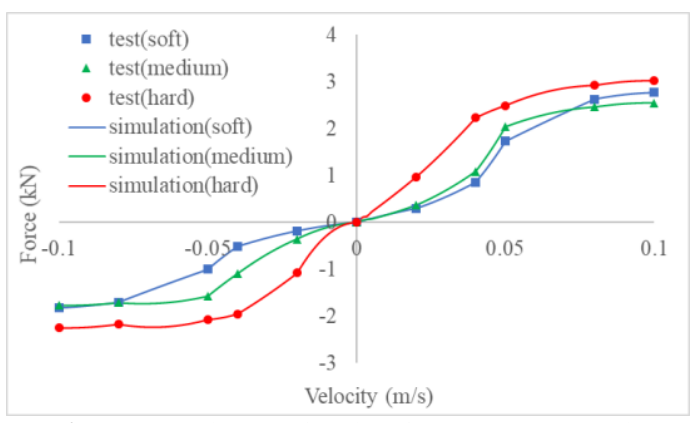

Figure 6. Comparison of axial force-compression speed relationship of the shock absorber between test and simulation

\subsection{Seat foam modeling}

The seat foam which is commonly made of low density polyurethane shows viscoelastic and hysteresis behaviors, respectively the strain rate dependency and the strain energy dissipation. The standard test method for characterizing these mechanical properties of PU foam are well documented in the literature (VSS manual, 2008). The numerical process of solving the mechanical interaction between seat foam and occupant by using a classical finite element analysis has been fully established and many successful applications have been reported for seat comfort design (Kim, 2007). On the other hand, a more analytic approach is proposed for a lumped network system modeling in this study. Figure 7 shows a stepwise and iterative process: 1) calculate strain and strain rate from the "Disp" element, 2) retrieve stress by making an interpolation Strain rate "curve family", 3) calculate the reaction force by applying a scale factor that is equivalent to the contact area between occupant and seat foam, 4) iterate 1-3 steps until reaching equilibrium.

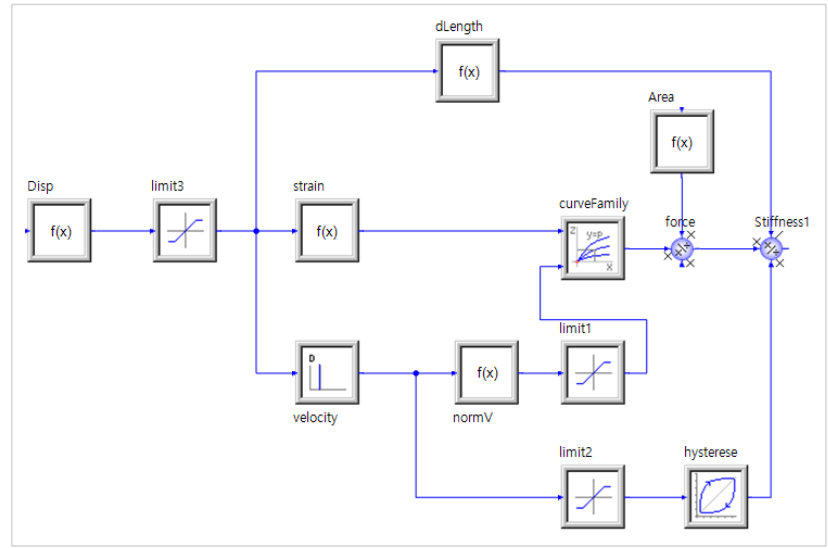

Figure 7. Process for structural analysis of seat foam in a lumped network system modeling

\section{Validation of air ride seat system modeling}

A two-stage excitation real test was conducted to validate the modeling of the air ride seat. As the first test, the dynamic characteristics of underbody part of the frame, mainly the reaction force produced by two parts 
in parallel connection, i.e., the air spring and the shock absorber, was identified by applying a harmonic excitation at the mounting floor. It was followed by the additional subcomponent serially attached on the top of underbody, the viscoelastic PU foam layer. Both excitation tests were conducted with a $75 \mathrm{~kg}$ dead weight placed on the top as representing a body weight of the occupant. The air inlet pressure was maintained at $9 \mathrm{bar}$ $(900 \mathrm{kPa})$ as same as the target large trailer in operation. The assembled 1D subcomponent models in the underbody frame introduced at sections 2.1 to 2.4 were validated using the $1^{\text {st }}$ stage test result. The $2^{\text {nd }}$ stage test was subsequently utilized for the model validation of the cushion foam in section 2.5.
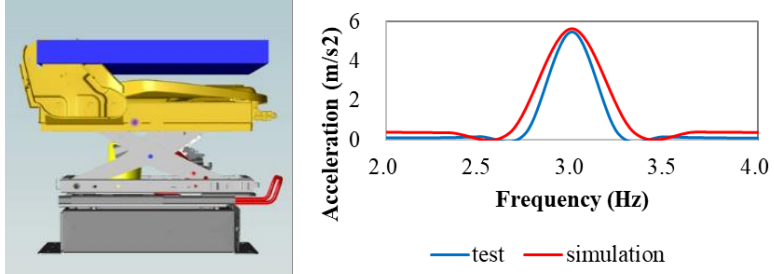

Figure 8. Harmonic excitation of underbody frame model with $75 \mathrm{~kg}$ dead weight

Figure 8 shows the underbody frame model with the $75 \mathrm{~kg}$ dead weight on the top, which was used for the simulation of sinusoidal harmonic excitation with $15 \mathrm{~mm}$ amplitude and $3 \mathrm{~Hz}$ speed. In the test, the dynamic response at the dead weight was measured by accelerometer sensor (Shimmer, Shimmer3 IMU Unit) with $256 \mathrm{~Hz}$ sampling rate. The Fast Fourier Transformation (FFT) signal shows a bell shape acceleration profile of the dead weight with $5.6 \mathrm{~m} / \mathrm{s}^{2}$ at $3 \mathrm{~Hz}$ as also shown in Figure 8 . The simulation result showed a bit wider bell shape than at the test but well correlated with the peak acceleration, $5.7 \mathrm{~m} / \mathrm{s}^{2}(+1.7 \%)$.

The modeling of seat foam was also validated by adding the subcomponent model described in section 2.5 on the top of the frame model. In the $2^{\text {nd }}$ stage test, a sine sweep excitation from $1 \mathrm{~Hz}$ to $20 \mathrm{~Hz}$, the same $75 \mathrm{~kg}$ dead weight was used but with the buttock shape of HPM (HPoint Machine). This buttock shape weight was allowed to move only along the vertical direction. The individual amount of contributions for the vibration damping by air suspension in the frame and the viscoelastic PU foam pad was separately analyzed. The transfer function from the air suspension which is the FFT profile of acceleration ratio between the floor and the top of the frame, shown in Figure 9 (a) indicated that the resonant frequency was around $2-3 \mathrm{~Hz}$ and overall transfer function was below 1.0. The other transfer function that was from the PU foam pad, the acceleration ratio between the top surface of the frame and buttock shape weight, shown in Figure 9 (b) revealed that its resonant frequency was around $6-7 \mathrm{~Hz}$ and the transfer function was above 1.0 up to $10 \mathrm{~Hz}$. The combined transfer function of the air ride seat system, the acceleration ratio between the floor and the buttock shape weight, is shown in Figure 9 (c). The summed total resonant frequency of the buttock shape weight against the floor vibration was $2 \mathrm{~Hz}$ and the transfer function rapidly declined after $8 \mathrm{~Hz}$, which was mainly due to the relatively low transfer function induced by the air suspension. Simulation result also in Figure 9 exhibits the same trend found at the test. However, there were some difference between the actual test and the system model also. There are nonlinear factors in the actual seats of commercial trucks, such as friction and tolerances, but they have not yet been covered in this study. They will consider in the next study. It is noteworthy that this kind of vibration damping performance at the air ride seat is quite distinctive in comparison with the one at the sedan seat. (Amann, 2008)

(a) between the floor and the frame

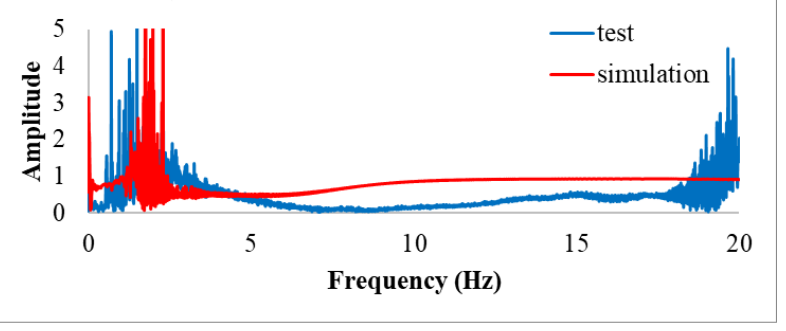

(b) between the frame and the buttock

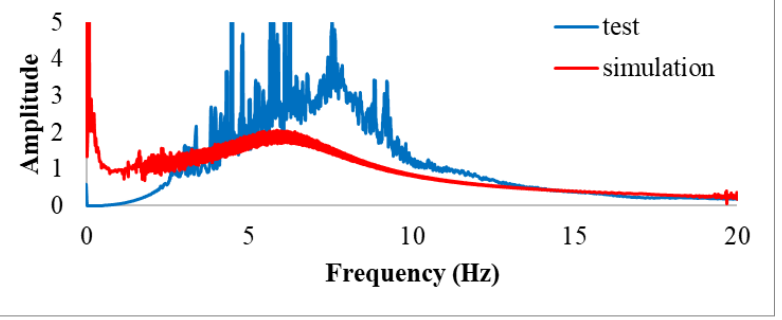

(c) between the floor and the buttock

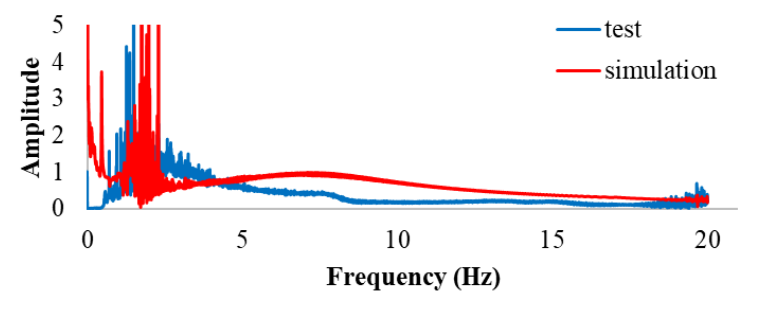

Figure 9. Harmonic excitation of air ride seat system with $75 \mathrm{~kg}$ buttock shape weight

\section{Verification of air ride seat system modeling}

In order to verify the practical use of the 1D modeling of air ride seat for the assessment of its comfort design, the international standard ISO 2631 is adopted, which determines the effect of vibrations on the human body (ISO 2631 part1, 1985). ISO 2631 contains methods for evaluating average comfort which has been widely used by researchers and vehicle designers. The quantified 
performance measures of ISO 2631 are based on frequency weighted root mean square, RMS, computations of acceleration data in accordance with the following equation

Where

$$
a_{w}=\left[\frac{1}{T} \int_{0}^{T} a_{w}^{2}(t) d t\right]^{\frac{1}{2}}
$$

$\mathrm{a}_{\mathrm{w}}(\mathrm{t})$ is the weighted acceleration (translational or rotational) as a function of time (time history), in meters per second squared $\left(\mathrm{m} / \mathrm{s}^{2}\right)$ or radians per second squared $\left(\mathrm{rad} / \mathrm{s}^{2}\right)$, respectively;

$\mathrm{T}$ is the duration of the measurement, in seconds.

The crest factor, defined as the modulus of the ratio of the maximum instantaneous peak value of the frequency-weighted acceleration signal to its RMS value, may be used to investigate if the incoming vibration contains occasional shocks or not in relation to its effects on human occupant. For vibration with crest factors below or equal to 9 , the basic evaluation method for the comfort score is normally sufficient. The ISO 2631 provides weighting function table. All necessary signal filters for the calculation of crest factor and ISO 2631 comfort index from the seat vibration that is the transient output from the 1D lumped network solver are implemented in to a total weighting function by using the "transfer function element" of Simulation $\mathrm{X}$ as shown in Figure 10.

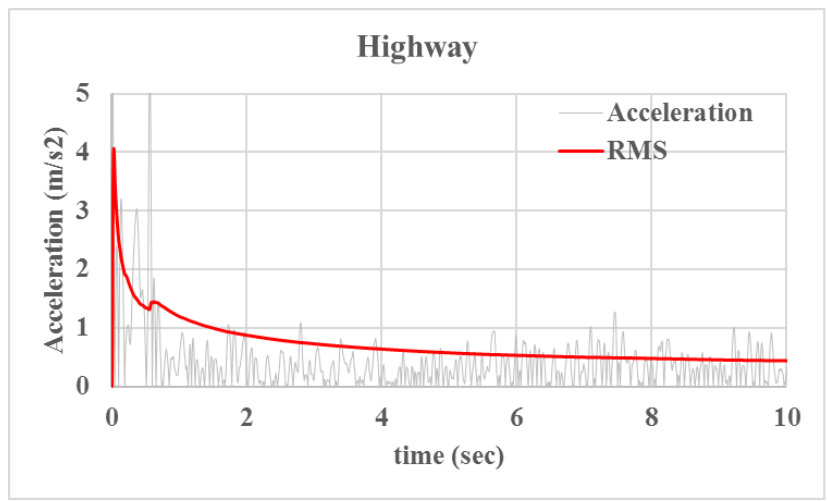

Total weighting function in ISO 2631

$$
\begin{aligned}
& H(p)=H_{h}(p) \cdot H_{l}(p) \cdot H_{t}(p) \cdot H_{s}(p) \\
& H_{h}(p): \text { High pass } \\
& H_{l}(p): \text { Low pass } \\
& H_{t}(p): \text { Acceleration-velocity transition } \\
& H_{s}(p): \text { Upward step }
\end{aligned}
$$$$
\times \mathrm{G}(\mathrm{s}) \quad G(s)=\frac{a_{0}+a_{1} \cdot s+a_{2} \cdot s^{2}}{b_{0}+b_{1} \cdot s+b_{2} \cdot s^{2}}
$$

e.g. Low pass

$$
\begin{aligned}
& \left|H_{h}(p)\right|=\left|\frac{1}{1+\frac{\sqrt{2} \omega_{1}}{p}+\left(\frac{\omega_{1}}{p}\right)^{2}}\right| \\
& G(s)=\frac{0+0 \cdot s+1 \cdot s^{2}}{\omega_{1}^{2}+\sqrt{2} \omega_{1} \cdot s+1 \cdot s^{2}}
\end{aligned}
$$

\begin{tabular}{|c|c|c|c|}
\hline Range & Index & Highway & Rough road \\
\hline Less than $0.315 \mathrm{~m} / \mathrm{s}^{2}$ & not uncomfortable & \multirow{6}{*}{0.4} & \multirow{6}{*}{1.1} \\
\hline $0.315 \mathrm{~m} / \mathrm{s}^{2}$ to $0.63 \mathrm{~m} / \mathrm{s}^{2}$ & a little uncomfortable & & \\
\hline $0.5 \mathrm{~m} / \mathrm{s}^{2}$ to $1 \mathrm{~m} / \mathrm{s}^{2}$ & fairly uncomfortable & & \\
\hline $0.8 \mathrm{~m} / \mathrm{s}^{2}$ to $1.6 \mathrm{~m} / \mathrm{s}^{2}$ & uncomfortable & & \\
\hline $1.25 \mathrm{~m} / \mathrm{s}^{2}$ to $2.5 \mathrm{~m} / \mathrm{s}^{2}$ & very uncomfortable & & \\
\hline Greater than $2 \mathrm{~m} / \mathrm{s}^{2}$ & extremely uncomfortable & & \\
\hline
\end{tabular}

Figure 10. Transfer function for ISO2631

Two kinds of measured floor acceleration time histories are applied for an exploratory operation of the 1D modeling of the air ride seat for the ISO 2631.

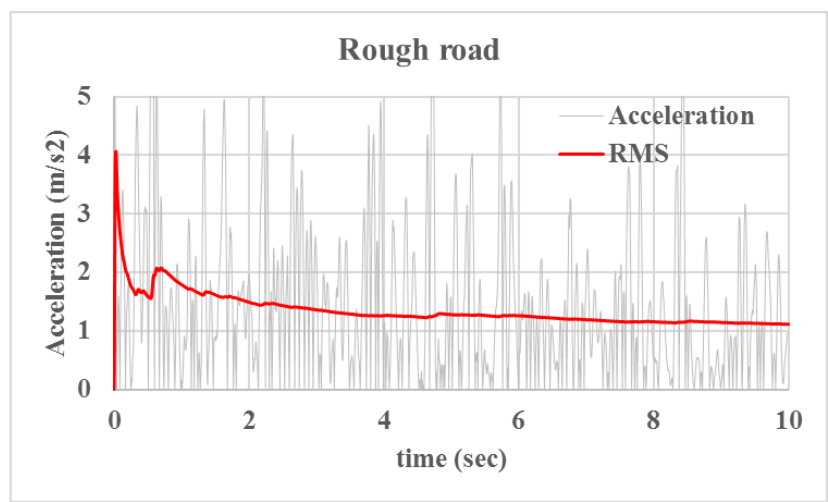

Figure 11. Room mean square acceleration of the buttock mass; the Highway(left) and the Rough road(right) condition

Table 1. ISO 2631 Comfort index 
Test driving was done with the target large truck at high speed proving track (hereafter called highway) and rough pavement test road (hereafter called rough road), respectively at $100 \mathrm{kph}$ and $30 \mathrm{kph}$ driving speeds. The 10 -second clip in the middle of floor accelerations measured at the driver side seat mounting point was used as an excitation input of the 1D air ride seat model. The same configuration of the buttock shape weight in the Figure 9 gauged the vibration response of the air ride seat model. The crest factors for the highway and the rough road were respectively 1.6 and 3.5, well below 9.0 and thus applicable to the assessment of comfort index. The computed RMS of frequency weighted filtered accelerations, i.e., the comfort score of highway driving was $0.44 \mathrm{~m} / \mathrm{s}^{2}$ and $1.11 \mathrm{~m} / \mathrm{s}^{2}$ for the rough road driving. They respectively belong to "a little uncomfortable" and "uncomfortable" categories. (see the Figure 11 and Table 1)

The effects of three-stage damping forces at the shock absorber, hard, medium and soft, on the ISO 2631 comfort index for both driving over the highway and rough road are evaluated. As the damping force increases to the hard stage from the medium, the comfort score was improved by $3.6 \%$ and $15 \%$ respectively at rough road driving (1.12->1.07) and high-speed PG driving (0.44->0.37). However, the decrease of damping force to the soft stage results in raises of RMS by $1.0 \%$ and $6.8 \%$. (see the Figure 12) As discussed in Section 2.3 , the amount of damping force change between hard and medium is more than the one between medium and soft stages and this brings more substantial reduction of RMS at hard stage which is recommendable in the aspect of transfer function of the buttock. This analysis does not include interactions with seat back and foot supports but just limited to between seat cushion and buttock.

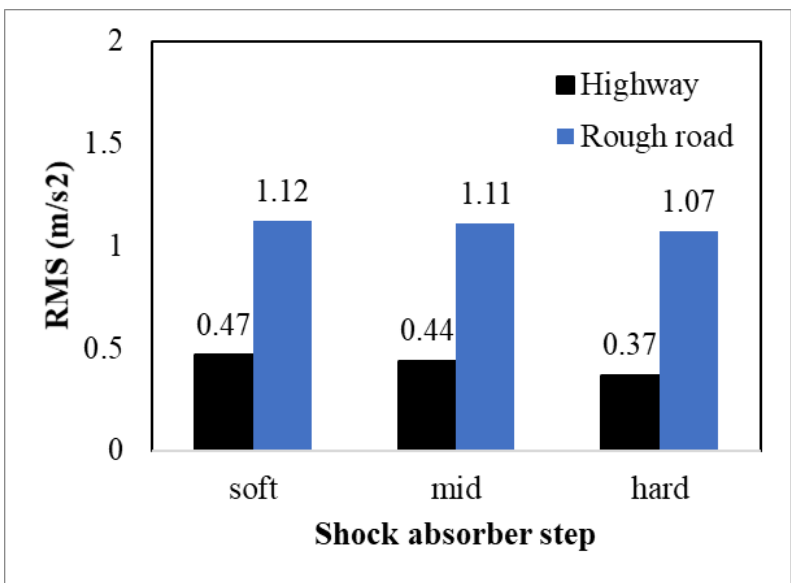

Figure 12. ISO 2631 comfort index between the highway and the rough road along the shock absorber

\section{Conclusions}

The air ride seat in a heavy-duty vehicle has multiphysical subcomponents such as air spring, shock absorber, and viscoelastic PU foam pad. The 1D lumped network modeling can be effectively used at the frontloading phase to optimize its engineering design parameters taking the relevant dynamic performance of neighboring parts into account. The mechanical characteristics of each major component in-vitro was experimentally identified and the corresponding 1D model was accordingly validated. The assembly of multi-physical subcomponents into the air ride seat was also validated against the harmonic excitation with a good correlation of the transfer function. In order to verify the practical application of the 1D air ride seat model for a ride comfort assessment, the standard ISO 2631 process was carried out for evaluation of each driving over the highway and the rough road. The effects of damping levels of shock absorber on the comfort score were also investigated.

The current validation and verification of 1D air ride model have a limitation such that a simple buttock shape weight is used as the occupant surrogate model. So the mechanical interactions are restricted to the seat cushion but no seat back and foot support are included. A virtual human body model with anatomically detailed joint articulation will be adopted in a subsequent study. Then, the scope of this ride comfort analysis will further expand to more precise interactions of the seat with the occupant model via deformable upholstery that may need a 3D mesh based solution, the finite element analysis. Therefore, the co-simulation of Functional Mockup Interface (FMI), in which the master and slave solutions are respectively $1 \mathrm{D}$ lumped network and 3D finite element analyses is also in our future plan for predicting a complete occupant kinematics for riding comfort simulation.

\section{References}

Mayton AG, DuCarme JP, Jobes CC, Matty TJ. ASME. Laboratory Investigation of Seat Suspension Performance During Vibration Testing., 2006. doi:10.1115/IMECE200614146

Yoo W.S., Park S.J., Park D.W., Kim Min-Seok, Lim O.K., Jeong W.B... International Journal of Automotive Technology. Comparison of ride comforts via experiment and computer simulation. 7. 309-314., 2006

Christian Amann, Andre Huschenbeth, Raphael Zenk, Nicole Montmayeur, Christian Marca, Carole Michel. Digital Human Modeling Symposium. Virtual Assessment of Occupied Seat Vibration Transmissibility, 2008. doi: 10.4271/2008-01-1861

Hao Liu, Jaecheon Lee, KSAE. An Experimental Investigation on the Characteristics of automotive air spring, pp. 4-8, 2008 
H.Y. Choi, K. Kim, C. Kim, S. Sah, S. Kim, S. Hwang, K. Lee, J. Pyun, N. Montmayeur, I. Lee. Digital Human Modeling Symposium. Challenge of Lumbar Support Design Using Human Body Models, 2008. doi: 10.4271/2008-01-1860

International Standard Organization ISO 2631/1. Evaluation of Human Exposure to Whole-body Vibration, Part1: General Requirements, 1985

N. Montmayeur, C. Marca, E. Haug, H.Y. Choi, S. Sah. Digital Human Modeling Symposium. Numerical and Experimental Analyses of Seating and Riding Comfort, 2004

S. Kim, S. Hwang, K. Lee, J. Pyun, H.Y. Choi, K. Kim, S. Sah, N. Montmayeur. Digital Human Modeling Symposium. New Anthropometry of Human Body Models for Riding Comfort Simulation, 2007. doi:10.4271/2007-01-2457

VSS manual, ESI GROUP SOLVER REFERENCE MANUAL. GETTING STARTED WITH PAM-COMFORT, page 18,2010

Whe-Ro Lee, Manyong Han, Hyung Yun Choi, Jungtae Yang, Inhyeok Lee, Kee Young Yang, Jong-Chan Park. PAM User Conference Asia. Air Ride Seat for Heavy Duty Vehicle. 2017 